\title{
O Método de Deformação como ferramenta didática na Teoria de Campos
}

The Deformation procedure as a didactic tool in the Field Theory

\author{
M. A. M. Souza*1] ${ }^{*}$ J.J. Rodrigues ${ }^{2}$ \\ ${ }^{1}$ Instituto Federal do Piauí, Parnaíba, PI, Brasil \\ ${ }^{2}$ Universidade Estadual da Paraíba, Araruna, PB, Brasil
}

Recebido em 26 de Dezembro, 2017. Revisado em 13 de Abril, 2018. Aceito em 12 de Junho, 2018.

\begin{abstract}
O método de deformação tem gerado inúmeros resultados satisfatórios dentro da teoria de campos, uma vez que o mesmo fornece uma maneira simples e até mesmo didática de descrever a estrutura de defeitos topológicos em diversos cenários. Ele consiste em gerar novos modelos a partir do potencial e da solução de um modelo conhecido, com o auxílio de uma função deformadora apropriada, gerando soluções analíticas sem precisar recorrer a métodos computacionais ou análise numérica. Neste trabalho demonstramos as diversas aplicações do método de deformação na teoria de campos escalares reais, estendendo-se desde a obtenção de potenciais polinomiais passando por modelos de dinâmica periódica até, resolução de equações diferencias não-lineares e o estudo de modelos de inflação cósmica no regime slow-roll.
\end{abstract}

Palavras-chave: método de deformação, função deformadora, campos escalares, equações diferencias não-lineares, inflação cósmica.

\begin{abstract}
The deformation method has generated many satisfactory results within the field theory, since it provides a simple and even didactic way of describing the structure of topological defects in several scenarios. It consists of generating new models from the potential and solution of a known model, with the aid of an appropriate deformation function, generating analytical solutions without having to resort to computational methods or numerical analysis. In this work, we demonstrate the different applications of the deformation method in real scalar field theory, ranging from the obtaining of potential polynomials through periodic dynamics models until, solving nonlinear differential equations and the study of cosmic inflation models in the slow-roll regime.

Keywords: deformation method, deformation function, scalar field, nonlinear differential equations, cosmic inflation.
\end{abstract}

\section{Introdução}

Em consequência do avanço das pesquisas em sistemas físicos, seja do ponto de vista fenomenológico seja do ponto de vista teórico, uma classe inumerável de modelos de campos escalares reais tem sido descoberta nas mais diversas áreas. Como exemplo, podemos citar que algumas das vertentes mais modernas da Cosmologia utilizam o campo escalar para descrever o campo do inflaton 1,4], que seria responsável pela aceleração cósmica do universo na sua fase inicial, ou ainda, poderíamos falar de modelos de quintessência [5 8 que atribuem a energia escura aspectos da dinâmica de campos escalares na fase atual do Universo.

Temos também em matéria condensada a descrição de defeitos topológicos em meios elásticos contínuos, associados a irregularidades nos campos que descrevem a ordenação da estrutura dos materiais. Esses defeitos surgem da quebra de simetria, que levam a uma transição de fase do sistema, e podem ser vórtices, kinks, paredes

*Endereço de correspondência: msouza@ifpi.edu.br de domínio, etc. O estudo de defeitos topológicos valeu o prêmio Nobel de Física de 2016 para os pesquisadores David Thouless, Duncan Haldane e Michael Kosterlitz.

Modelos de campos escalares descritos por potenciais trigonométricos, como o seno-Gordon e duplo senoGordon, aparecem em inúmeros contextos físicos, muitas de suas aplicações residem na modulação de sinais elétricos em circuitos de corrente alternada [9], em cadeias moleculares poliméricas compostas por nucleotídeos, como o DNA 1012 e no estudos de impedância em filmes de materias inorgânicos [13], entre outras.

Entretanto, a dificuldade reside no fato que muito dos potenciais desses modelos não propiciam uma descrição analítica do sistema investigado, o que dificulta o entendimento mais completo das propriedades de tais modelos. Torna-se, portanto, necessário buscar-se um método que possa gerar potenciais com soluções analíticas e de interesse físico. Um método bastante eficaz é o denominado método de deformação, cujo procedimento consiste em gerar novos modelos a partir do potencial e da solução de um modelo conhecido, com o auxílio de uma função 
deformadora apropriada $14-18$. A vantagem do método está no fato de que a descrição das características do novo modelo pode ser feita de forma analítica sem precisar recorrer a simulações computacionais ou análise numérica. Sendo também aplicado na resolução de equações diferencias não-lineares em sistemas integráveis.

Partindo da perspectiva que uma metodologia é considerada eficaz didaticamente se a mesma for consistente, prática e eficiente em produzir fácil entendimento de um tema visto como complexo, podemos afirmar que o método de deformação fornece uma abordagem didática de vários sistemas de interesse físico, pois permite descrever propriedades de modelos ou fenômenos desconhecidos de forma analítica e simples, bastando para isso conhecer soluções de um modelo ou equação padrão já estabelecidos.

Neste trabalho iremos abordar o método de deformação em vários cenários de interesse no meio cientifico, visando mostrar que o mesmo é uma ferramenta didática extremamente eficaz na descrição e entendimento de diversos sistemas físicos. Na seção II demonstraremos como funciona o método, em seguida na seção III, a partir do método, descreveremos a estrutura de defeitos topológicos em modelos regidos por potenciais polinomiais, na seção IV vamos estudar a dinâmica de modelos do tipo seno-gordon, na seção $\mathrm{V}$ mostraremos o procedimento de resolução de equações diferencias não-lineares do tipo KdV, na seção VI demonstraremos as aplicações na geração de modelos cosmológicos inflacionários e as considerações finais serão feitas na seção VII.

\section{O Método de deformação na teoria de campos}

O método de deformação de defeitos foi proposto em 14.15. Partimos de um potencial $V(\phi)$ conhecido que possui soluções com topologia definida, desse modo podemos definir um novo modelo cujas propriedades podem ser obtidas através do modelo primitivo. É notório salientar a eficácia de tal método que possibilita uma descrição analítica de uma ampla classe de potenciais, por meio de sucessivas deformações, levando em conta o ajuste adequado dos parâmetros de uma função deformadora conveniente. Suas aplicações de estendem até a resolução de equações não lineares, que descrevem fenômenos ondulatórios como a equação KdV 19,20. Inicialmente vamos estabelecer a relação entre o potencial do modelo primitivo e o potencial do modelo obtido através da deformação. Sabemos que a densidade lagrangeana para o campo escalar real $\phi$ é dada por:

$$
\mathcal{L}=\frac{1}{2} \partial_{\mu} \phi \partial^{\nu} \phi-V(\phi),
$$

que para soluções estáticas, $\phi=\phi(x)$, fornece a equação de movimento:

$$
\frac{d^{2} \phi}{d x^{2}}=\frac{d V}{d \phi},
$$

que é resolvida pelas equações de primeira ordem:

$$
\frac{d \phi}{d x}= \pm \sqrt{2 V(\phi)} .
$$

Se tomarmos outro modelo, descrito por um campo $\chi$, com densidade lagrangeana dada por:

$$
\mathcal{L}=\frac{1}{2} \partial_{\mu} \chi \partial^{\mu} \chi-\tilde{V}(\chi),
$$

temos, para a solução estática, $\chi=\chi(x)$, a equação de movimento:

$$
\frac{d^{2} \chi}{d x^{2}}=\frac{d \tilde{V}}{d \chi}
$$

soluvel pelas equações de primeira ordem:

$$
\frac{d \chi}{d x}= \pm \sqrt{2 \tilde{V}(\chi)} .
$$

Se considerarmos $\phi=f(\chi)$, isto é, $\phi$ como uma função de $\chi$ temos:

$$
\frac{d \phi}{d x}=\frac{d f}{d \chi} \frac{d \chi}{d x} \Rightarrow \frac{d \chi}{d x}=\frac{1}{d f / d \chi} \frac{d \phi}{d x},
$$

elevando a segunda equação de (7) ao quadrado temos:

$$
\left(\frac{d \chi}{d x}\right)^{2}=\frac{1}{f_{\chi}^{2}}\left(\frac{d \phi}{d x}\right)^{2}
$$

onde $f_{\chi}=d f / d \chi$. Substituindo as equações (2) e (6) na equação (8) obtemos:

$$
\tilde{V}(\chi)=\frac{V[\phi=f(\chi)]}{f_{\chi}^{2}} .
$$

A função $f(\chi)$ é chamada função deformadora. A expressão (9) nos dá a relação entre os potenciais no modelo deformado e não deformado. O potencial $\tilde{V}$ admite soluções,que são obtidas pela relação:

$$
\chi(x)=f^{-1}(\phi(x)),
$$

em que $f^{-1}$ é a inversa da função deformadora. Ainda podemos ver que, para o caso de soluções topológicas o defeito deformado $\chi(x)$ conecta mínimos correspondentes aos interligados pelas soluções $\phi(x)$ do modelo original dados por $\tilde{v}_{i}=f^{-1}\left(v_{i}\right), i=1,2,3 \ldots, n$.

\subsection{Propriedades do Método}

Uma particularidade do método está associada a deformação do modelo $\phi^{4}[21]$ dado por:

$$
V(\phi)=\frac{1}{2}\left(1-\phi^{2}\right)^{2} .
$$

Se utilizarmos uma função deformadora $f(\chi)$ na obtenção de um modelo deformado, a função $1 / f(\chi)$ também irá gerar o mesmo modelo deformado. A prova desta afirmativa pode ser verificada como segue: seja a função 
deformadora $f_{1}=F(\chi)$, aplicada ao potencial (11), que leva ao potencial deformado:

$$
\tilde{V}_{1}(\phi)=\frac{1}{2} \frac{\left(1-F(\chi)^{2}\right)^{2}}{F_{\chi}^{2}} .
$$

Considere agora a função deformadora $f_{2}=1 / F(\chi)$, também aplicada em (11), de forma que obtemos o novo potencial deformado dado por:

$$
\begin{aligned}
\tilde{V}_{2}(\phi) & =\frac{1}{2}\left(1-\frac{1}{F(\chi)^{2}}\right)^{2} \frac{F(\chi)^{4}}{F_{\chi}^{2}} \\
& =\frac{1}{2} \frac{\left(1-F(\chi)^{2}\right)^{2}}{F_{\chi}^{2}}
\end{aligned}
$$

$\log \mathrm{O}$

$$
\tilde{V}_{2}(\phi)=\tilde{V}_{1}(\phi),
$$

como queriamos demonstrar. Outro resultado interessante versa sobre a relação entre a derivada segunda do potencial para o modelo original e o modelo deformado calculada nos mínimos. Fisicamente, o que estamos fazendo é determinar a relação que existe entre a massa quadratica do campo nos dois modelos. Seja o potencial dado por (9), vamos deriva-lo em relação a $\chi$ duas vezes, de modo que obtemos o resultado:

$$
\begin{array}{r}
\tilde{V}_{\chi \chi}(\chi)=V_{\phi \phi}(\phi=f(\chi))-\frac{3 V_{\phi}(\phi=f(\chi)) f_{\chi \chi}}{f_{\chi}^{2}}+ \\
+\frac{6 V(\phi=f(\chi)) f_{\chi \chi}^{2}}{f_{\chi}^{4}}-\frac{2 V(\phi=f(\chi)) f_{\chi \chi \chi}}{f_{\chi}^{3}}
\end{array}
$$

para os pontos de mínimo ou estados de vácuo comuns aos dois modelos temos que o potencial e sua derivada primeira são nulos, $V=V_{\phi}=0$, de modo que encontramos o resultado final:

$$
\tilde{V}_{\chi \chi}(\chi)=V_{\phi \phi}(\phi=f(\chi)),
$$

que mostra que a massa quadratica do campo, no modelo original e no modelo deformado são iguais quando calculadas nos mínimos comuns aos dois modelos.

\subsection{Estados BPS no modelo deformado}

Um método alternativo para investigar a presença de defeitos topológicos em modelos de campos escalares, consiste em identificar as soluções denominadas de BPS (Bogomol'nyi-Prasad-Sommerfield) 22,23]. As soluções BPS são definidas por dois estados de mínima energia e identificam os setores topológicos do modelo. O método foi proposto por E.B. Bogomol'nyi em 1976, embora em 1975 M.K. Prasad e C.M. Sommerfield já tivessem publicado um trabalho sobre o assunto. A idéia central está no fato de que para potenciais não negativos, cujos zeros são mínimos globais, podemos escrever:

$$
V(\phi)=\frac{1}{2} W_{\phi}^{2}
$$

onde a derivada de $\mathrm{W}$ em relação a $\phi$, representada por $W_{\phi}$, e $W(\phi)$ são funções contínuas do campo, sendo esta ultima denominada superpotencial e está associada a descrição de setores bosônicos reais em teorias supersimetricas 24 26]. Para o modelo $\phi^{4}$, temos:

$$
W(\phi)=\phi-\frac{1}{3} \phi^{3} \text {. }
$$

A equação de movimento para soluções estáticas é dada por:

$$
\frac{d^{2} \phi}{d x^{2}}=W_{\phi} W_{\phi \phi}
$$

As soluções fisicamente aceitáveis são as de energia finita, as configurações de menor energia são as de maior interesse para nossa descrição, logo o campo deve ser escolhido de modo que a energia $E_{B}$ seja mínima, esta ultima é também conhecida como energia de Bogomol'nyi, dada por:

$$
\begin{aligned}
E_{B} & =\int_{-\infty}^{\infty} d x \frac{d W}{d x} \\
& =W(\phi(x \rightarrow \infty))-W(\phi(x \rightarrow-\infty)) \\
& =|\Delta W| .
\end{aligned}
$$

Para a condição de energia mínima obtemos a equação de primeira ordem:

$$
\frac{d \phi}{d x}= \pm W_{\phi}
$$

que resolve a equação (19). E o problema de resolver uma equação de segunda ordem se reduz a encontrar as soluções de duas equações de primeira ordem [27,28]. A expressão 21) é conhecida na literatura como equação de Bogomol'nyi. Outra vantagem desse método é que podemos calcular as energias usando somente a função $W(\phi)$ por meio de seus limites assintóticos sem conhecer as soluções. Iremos agora estabelecer a relação entre os estados BPS no modelo original e no modelo deformado. Utilizando as equações (9) e (17), considerando ainda $\phi=f(\chi)$, se $\tilde{V} \geq 0$, podemos escrever para o potencial do sistema deformado:

$$
\tilde{V}(\chi)=\frac{1}{2}\left(\frac{W_{\phi}(\phi=f(\chi))}{f_{\chi}}\right)^{2},
$$

$\mathrm{Ou}$

$$
\tilde{V}(\chi)=\frac{1}{2}\left(\tilde{W}_{\chi}(\chi)\right)^{2}
$$

por comparação direta entre 22 e 23 obtemos:

$$
\tilde{W}_{\chi}(\chi)=\frac{W_{\phi}(\phi=f(\chi))}{f_{\chi}},
$$

que nos permite escrever as equações de primeira ordem:

$$
\frac{d \chi}{d x}= \pm \frac{W_{\phi}(\phi=f(\chi))}{f_{\chi}},
$$

nos possibilitando resolver as equações de movimento no modelo deformado. A energia para o defeito deformado 
depende diretamente do tipo de deformação introduzida, uma vez que podemos mostrar, com a ajuda de (10), que para os estados BPS temos:

$$
\begin{aligned}
\tilde{E}_{B P S} & =\int_{-\infty}^{\infty} d x\left(\frac{d \chi}{d x}\right)^{2} \\
& =\int_{-\infty}^{\infty} d x\left(\frac{d f^{-1}}{d \phi}\right)^{2}\left(\frac{d \phi}{d x}\right)^{2} .
\end{aligned}
$$

A densidade de energia no modelo deformado pode então ser escrita como:

$$
\tilde{\epsilon}(x)=\left(\frac{d f^{-1}}{d \phi}\right)^{2}\left(\frac{d \phi}{d x}\right)^{2}=\left(\frac{d f^{-1}}{d \phi}\right)^{2} \epsilon(x),
$$

onde $\epsilon(x)$ é a densidade de energia no modelo original. Outro ponto importante é que para uma classe de funções deformadoras $f(\chi)$ satisfazendo $|f(\chi)| \geq 1(\leq 1)$, a energia diminui(aumenta) de forma relativa a energia do defeito não deformado.

\subsection{Estabilidade dos defeitos deformados}

O estudo da estabilidade para as soluções BPS do modelo deformado é análogo ao do modelo original, que pode ser encontrado em [21,29], de modo que obtemos para a solução estática do campo a equação do tipo Schrödinger:

$$
\left(-\frac{d^{2}}{d x^{2}}+\tilde{U}(x)\right) \tilde{\eta}=\tilde{\omega}_{n} \tilde{\eta}
$$

em que

$$
\tilde{U}(x)=\left(\frac{d^{2} \tilde{V}}{d \chi^{2}}\right)_{\chi=\chi(x)} .
$$

O operador Hamiltoniano possui a forma:

$$
H=-\frac{d^{2}}{d x^{2}}+\tilde{U}(x)
$$

que pode ser fatorado em $H=\tilde{S}^{+} \tilde{S}$ com $\tilde{S}$ escrito em termos do super-potencial não deformado na forma:

$$
\tilde{S}=\frac{d}{d x}+W_{\phi \phi}(\phi=f(\chi))-f_{\chi \chi} \frac{W_{\phi}(\phi=f(\chi))}{f_{\chi}^{2}} .
$$

Uma vez que o Hamiltoniano pode ser fatorado o modelo apresenta soluções estáveis com os respectivos autovalores reais positivos. O modo zero bosônico deformado é dado por:

$$
\tilde{\eta}_{0}=A e^{ \pm \int \tilde{W}_{\chi \chi}(\chi)} d x
$$

onde A é uma constante de normalização. Reescrevendo esta ultima equação em função do modelo original, encontramos:

$$
\tilde{\eta}_{0}=\eta_{0} \exp \left[\int d x f_{\chi \chi} \frac{W_{\phi}(\phi=f(\chi))}{f_{\chi}^{2}}\right],
$$

em que $\eta_{0}$ é o modo zero bosônico do modelo não deformado. Outra forma de escrever 33 seria:

$$
\tilde{\eta}_{0}=A \tilde{W}_{\chi}
$$

que ainda pode ser posta na forma:

$$
\tilde{\eta}_{0}=A \frac{W_{\phi}(\phi=f(\chi))}{f_{\chi}} .
$$

Nas seções seguintes iremos aplicar o Método de deformação de forma geral dando destaque somente a obtenção dos potencias e suas soluções, de modo a não tornar a leitura deste trabalho extensa e enfadonha.

\section{Obtendo potenciais polinomiais}

Vamos verificar que alguns potenciais conhecidos na literatura podem ser obtidos pela deformação do modelo $\phi^{4}$, ver Figura 1, que tem soluções estáticas $\phi= \pm \tanh (x)$.

\subsection{Deformando modelos com defeitos do tipo kink}

Aplicando a função deformadora $\mathbf{f}(\chi)=\mathbf{2} \chi^{\mathbf{2}}-\mathbf{1}$, obtemos o potencial deformado ilustrado na Figura 2:

$$
\widetilde{V}(\chi)=\frac{1}{2} \chi^{2}\left(1-\chi^{2}\right)^{2},
$$

conhecido como o potencial do modelo $\phi^{6}$, e que possui os mínimos $\bar{\chi}= \pm 1$ e $\bar{\chi}=0$. Aqui, as soluções do modelo deformado são obtidas por meio da função deformadora inversa

$$
\chi(x)= \pm \sqrt{(1+\phi(x)) / 2},
$$

$\operatorname{com} \phi(x)= \pm \tanh (x)$,ou seja:

$$
\chi(x)= \pm \sqrt{[1 \pm \tanh (x)] / 2} .
$$

Para obter as soluções do modelo $\phi^{6}$ sem deformação teriamos que resolver a equação diferencial:

$$
\frac{d \chi}{d x}=\sqrt{2 \widetilde{V}}= \pm \chi\left(1-\chi^{2}\right)
$$

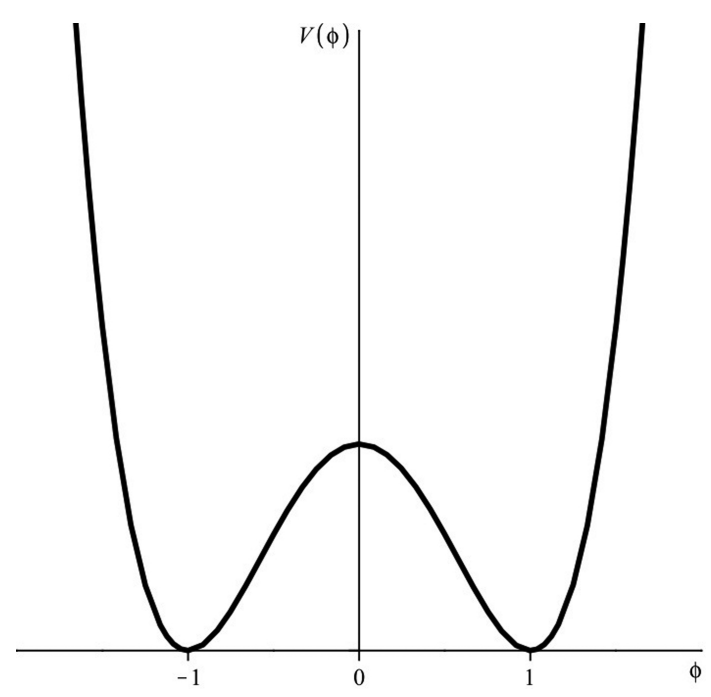

Figura 1: potencial do modelo $\phi^{4}$. 

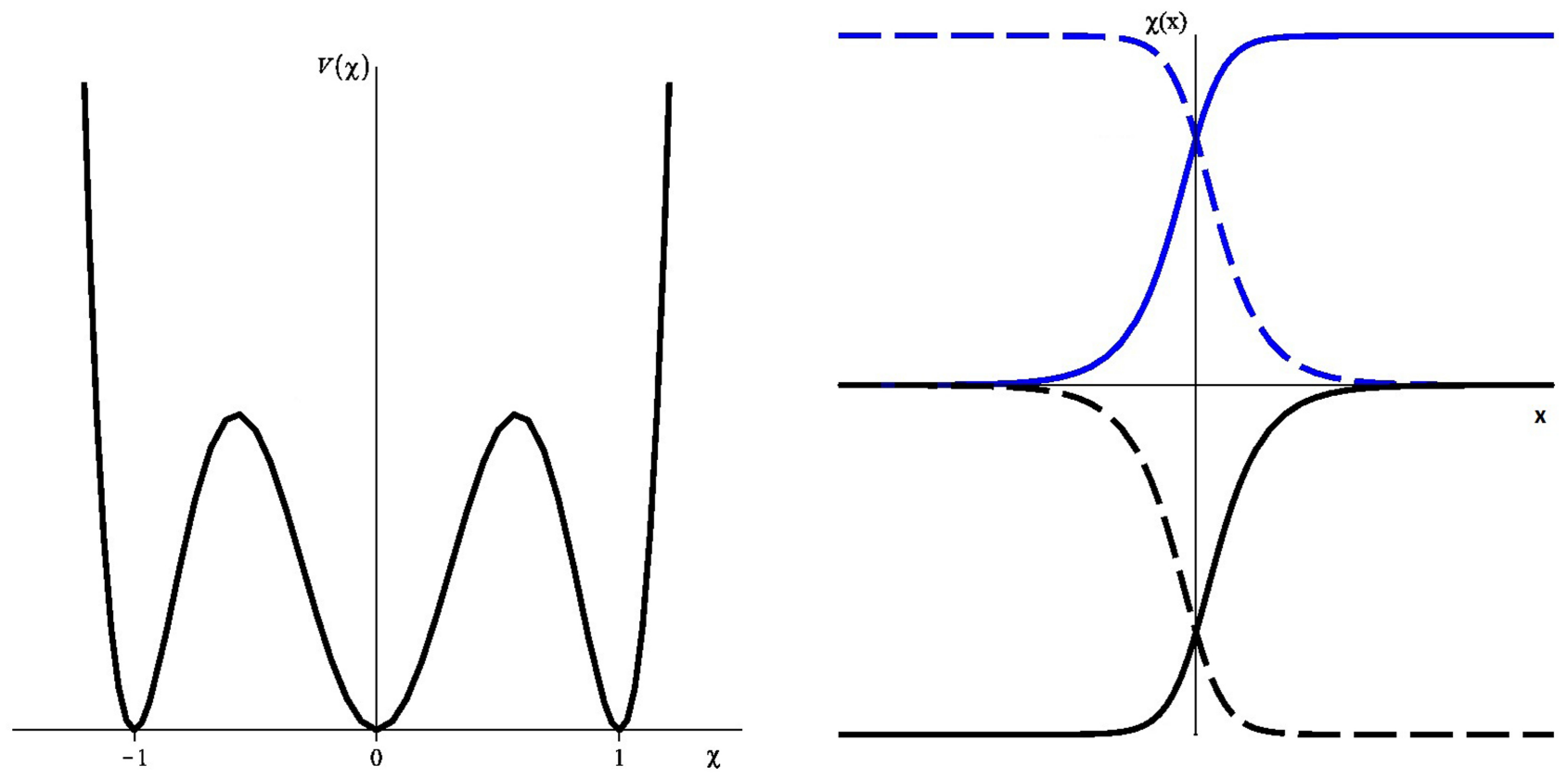

Figura 2: Potencial $\phi^{6}$ a esquerda e suas soluções kinks (linha sólida) e anti-kinks (linha tracejada) a direita.

que e dada pela inversa da integral

$$
\pm x=\int \frac{d \phi}{\chi\left(1-\chi^{2}\right)},
$$

o que ainda é exequível, porém, para potenciais polinomiais de grau mais elevado, geralmente isto é uma tarefa muito árdua, ou infactível, como por exemplo, para o potencial do modelo $\phi^{8}$ :

$$
V=\frac{1}{2}\left(1-\phi^{2}\right)^{2}\left(1-2 \phi^{2}\right)
$$

\section{Obtendo potenciais do tipo seno-Gordon}

Vimos, anteriormente, o método de deformação e suas aplicações em modelos de potenciais polinomiais com soluções do tipo kink. Agora, iremos estudar as propriedades de kinks em modelos de potenciais periódicos do tipo seno-Gordon. O interesse nesse tipo de modelo reside no fato do mesmo possuir inúmeras aplicações em Física de altas energias, assim como em Matéria condensada. Inicialmente, podemos estudar o modelo seno-Gordon como resultado da deformação do modelo $\phi^{4}$ pela função deformadora do tipo:

$$
f(\chi)=\operatorname{sen}(a \chi)
$$

de onde obtemos:

$$
V(\chi)=\frac{1}{2} \cos ^{2}(a \chi)
$$

O potencial dado por 43 é do tipo sine-Gordon, bem conhecido na literatura, ele foi inicialmente estudado por Rubinstein em 1968 [30], algumas de suas aplicações que se estendem desde sistemas com processamento de sinal elétrico ou mecânico até cadeias moleculares em sistemas biológicos podem ser encontradas em [9, 10]. Os mínimos do potencial são $\bar{\chi}=(2 n+1) \pi / 2 a, \operatorname{com} n$ inteiro positivo ou negativo $(n=0, \pm 1, \pm 2, \pm 3, \ldots)$. As soluções que conectam os mínimos do potencial, obtidas pela função deformadora inversa de 42 , são dadas por:

$$
\chi(x)= \pm \frac{1}{a} \operatorname{arcsen}(\tanh (x))+\frac{2 k \pi}{a}, \quad k=\text { inteiro. }
$$

\subsection{Modelo duplo seno-Gordon}

Vamos aplicar as funções deformadoras:

$$
\begin{gathered}
f_{1}(\chi)=r \tan (\chi), \\
f_{2}(\chi)=\frac{1}{r} \tan \left(\chi-\frac{\pi}{2}\right),
\end{gathered}
$$

onde $r$ é um parâmetro real e positivo, note que $f_{2}$ é igual a $1 / f_{1}$. Utilizamos duas funções deformadoras pois desejamos descrever um modelo com dois setores topológicos distintos. O potencial deformado pode ser escrito como:

$$
V(\chi)=\frac{\left(\cos ^{2}(\chi)-r^{2} \operatorname{sen}^{2}(\chi)\right)^{2}}{2 r^{2}},
$$

onde para $r>0$, exceto $r=1$, obtemos modelos duplo seno-Gordon [31], ver Figura 3.

O potencial (47) foi utilizado por Batanouny et al [13] em sistemas de Matéria Condensada, e algumas de suas propriedades termodinâmicas foram estudadas em [32]. Podemos ver que o parâmetro $r$ controla a posição dos 


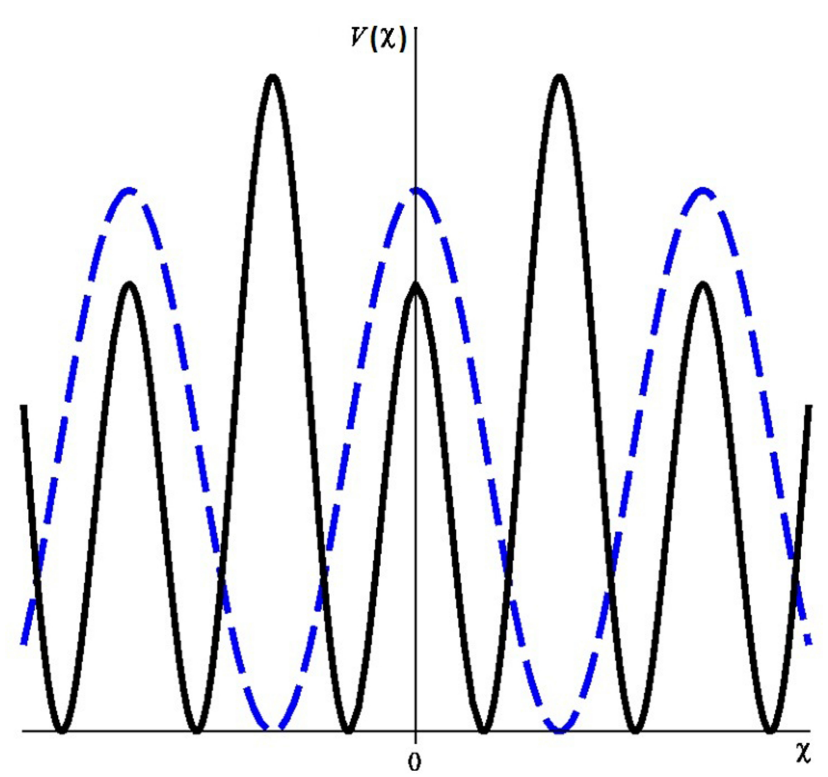

Figura 3: Potencial do modelo seno-Gordon (linha tracejada) e potencial do modelo duplo seno-Gordon (linha sólida) para $r=1,1$.

diferentes setores topológicos do modelo. Os mínimos do potencial são dados por:

$$
\chi_{m i n}^{n}=n \pi \pm \arctan (1 / r), \quad n=\text { inteiro. }
$$

Com este método pode-se gerar uma família de novos modelos do tipo seno-Gordon 33, 34.

\section{Sistemas integráveis: Equação $\mathrm{KdV}$}

O estudo de soluções de energia localizada foi iniciado por J. Scott Russel em 1834, quando este observou a propagação de um pulso de onda concentrado no canal de Edinburgh-Glasgow, que se propagou por uma elevada distância sem perder a forma original [35]. A Figura 4 ilustra o processo análogo de formação de ondas solitárias em um canal, também denominadas de solitons.

Em 1895 os matemáticos D.J.Korteweg e G. de Vries 36] propuseram uma equação que descreve o fenômeno observado por Russel. A equação KdV é utilizada para descrever a propagação de um pulso de onda não linear em um meio material, como também é aplicada no estudo de ondas na atmosfera, ondas íon-acústicas em um plasma e ondas de pressão em misturas de líquido e gases $37 \sqrt{42}$.

O estudo de solitons levou a um grande avanço na construção das fibras óticas 43, 44, fazendo as perdas de informação ao longo da fibra tornarem-se cada vez menores, diminuindo a dispersão dos pulsos luminosos ao longo da propagação da onda, como ilustrado na Figura 5 .

Matematicamente a equação KdV pode ser expressa como:

$$
u_{t}+6 u u_{x}+u_{x x x}=0,
$$

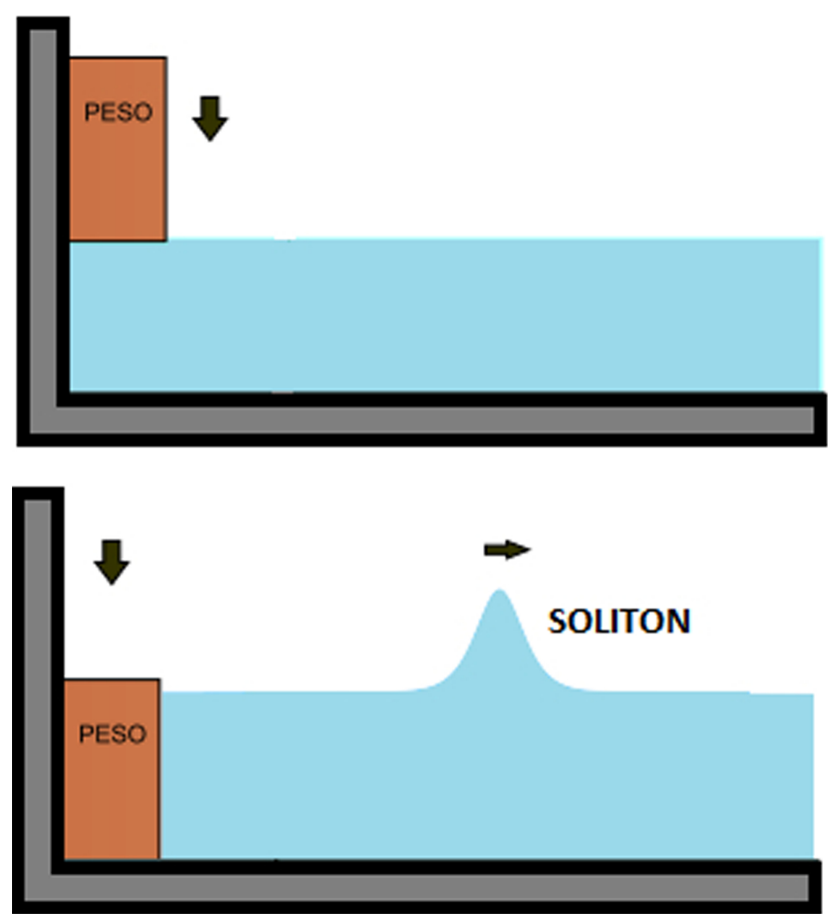

Figura 4: Processo de geração de ondas solitarias

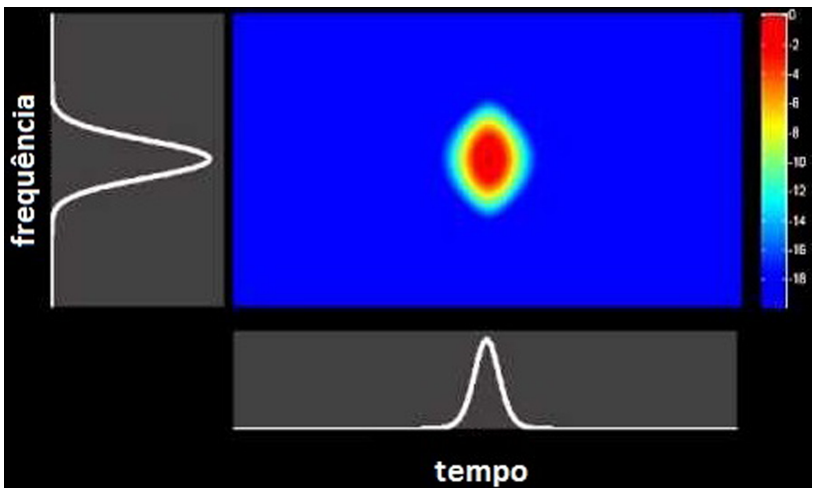

Figura 5: Simulação númerica do espectro de propagação de um soliton em fibra ótica. Créditos: Christophe Finot. Laboratoire Interdisciplinaire CARNOT de Bourgogne, Solitons, Lasers and Optical Communications Team.

onde:

$$
u_{t}=\frac{\partial u}{\partial t} \quad \text { e } u_{x}=\frac{\partial u}{\partial x} .
$$

A solução tipo onda viajante pode ser escrita na forma:

$$
u=2 k^{2} \operatorname{senh}^{2}\left(k x-4 k^{3} t\right)=\frac{c}{2} \operatorname{senh}^{2}(k x-c t),
$$

em que $c=\omega / k=4 k^{2}$ é a velocidade da onda. Considere agora um sistema dinâmico na variável $u=u(x, t)=u(z)$ descrito pela equação diferencial:

$$
u_{t}+(f(u))_{x}+u_{x x x}=0
$$

sendo $f(u)$ uma função da variável dinâmica $u$ [19], temos ainda que:

$$
(f(u))_{x}=\frac{\partial f}{\partial u} u_{x}
$$


Para soluções do tipo onda viajante $u=u(k x-\omega t)$, com $z=k x-\omega t$, obtemos as relações:

$$
u_{t}=-\omega u_{z} \text { e } u_{x}=k u_{z},
$$

resultando na equação:

$$
u_{t}=-\frac{\omega}{k} u_{x}
$$

A equação 52 torna-se:

$$
-\frac{\omega}{k} u_{x}+(f(u))_{x}+u_{x x x}=0,
$$

colocando a derivada em $x$ em evidência:

$$
\frac{d}{d x}\left(-\frac{\omega}{k} u+f(u)+u_{x x}\right)=0 .
$$

Integrando obtemos:

$$
u_{x x}=\frac{\omega}{k} u-f(u)+\beta,
$$

onde $\beta$ é uma constante de integração, que pode ser obtida das condições assintóticas. Podemos escrever a equação 58 na forma:

$$
\frac{d^{2} u}{d x^{2}}=\frac{d V(u)}{d u} .
$$

A função $V(u)$ é conhecida como potencial associado, é apenas uma ferramenta matemática, um fator de integração, não tendo o significado de um potencial físico de interação. Agora, tomando um mapa inversível com uma função deformadora do tipo:

$$
u=g(v),
$$

tal que:

$$
\tilde{V}(v)=\frac{V(g(v))}{\left(\frac{d g(v)}{d v}\right)^{2}},
$$

observamos que o modelo na variável dinâmica $v(x, t)$ descrito por:

$$
v_{t}+(\tilde{f}(v))_{x}+v_{x x x}=0,
$$

para o qual o potencial associado possa ser obtido de 61) e satisfaz a realação:

$$
\frac{d \tilde{V}(v)}{d v}=\frac{\tilde{\omega}}{k} v-\tilde{f}(v)+\tilde{\beta},
$$

tem como solução a onda viajante dada pelo mapa inverso:

$$
v(x, t)=v(k x-\tilde{\omega} t)=g^{-1}[u(k x-\omega t)] .
$$

Portanto, se a solução onda viajante do sistema (52) é conhecida, podemos obter através do mapa inverso a solução do sistema 62 19 20. É um método simples e direto para encontrar soluções tipo onda viajente em sistemas dinâmicos integráveis não-lineares, quando comparado aos demais métodos encontrados na literatura como o método do espalhamento inverso [38], metódo bilinear de Hirota 45], Representação de Lax [46], entre outros.

\subsection{Aplicação}

Vamos obter a solução tipo onda viajante do sistema descrito pela equação pKdV 19]:

$$
v_{t}+a(1+p)(1+2 p) v^{1 / p} v_{x}+v_{x x x}=0
$$

onde a e p são parâmetros reais e para $a=p=1$ obtemos a equação KdV. Temos ainda que

$$
f^{\prime}(v)=a(1+p)(1+2 p) v^{1 / p} .
$$

Por integração direta, conforme as equação (58) e 63), obtemos:

$$
\frac{d \tilde{V}(v)}{d v}=\frac{\tilde{\omega}}{k} v-a p(1+2 p) v^{\frac{1}{p}+1}+\tilde{\beta},
$$

onde $f(v)=a p(1+2 p) v^{\frac{1}{p}+1}$, destacando que $\tilde{\omega}$ e a constante de integração $\tilde{\beta}$ serão obtidas posteriormente. Partindo agora da equação KdV, cuja solução tipo onda viajante é dada por:

$$
u=2 k^{2} \operatorname{senh}^{2}\left(k x-4 k^{3} t\right),
$$

em que $\omega=4 k^{3}$, conforme as equações 58 e 59 obtemos o resultado:

$$
u_{x x}=\frac{d V}{d u}=\frac{\omega}{k} u-3 u^{2}+\beta,
$$

em que utilizamos $f^{\prime}(u)=6 u$, levando a $f(u)=3 u^{2}$. O valor de $\beta$ é zero e o potencial associado para $\mathrm{KdV}[19$ possui a forma:

$$
V(u)=2 k^{2} u^{2}-u^{3} .
$$

Tomando o mapa:

$$
u(k x-\tilde{\omega} t)=g\left[v(k x-\tilde{\omega} t]=a v^{1 / p},\right.
$$

e utilizando as equações 61 e 70 obtemos o potencial associado:

$$
\tilde{V}(v)=\frac{V(g(v))}{\left(\frac{d g(v)}{d v}\right)^{2}}=2 k^{2} p^{2} v^{2}-a p^{2} v^{\frac{1}{p}+2},
$$

derivando 72 em relação a $v$ e substituindo o valor da derivada na equação 67 encontramos a igualdade de polinômios:

$$
\frac{\tilde{\omega}}{k} v-a p(1+2 p) v^{\frac{1}{p}+1}+\tilde{\beta}=4 k^{2} p^{2} v-a p(1+2 p) v^{\frac{1}{p}+1},
$$

que leva a $\tilde{\beta}=0$ e $\tilde{\omega}=4 k^{3} p^{2}$. E o mapa inverso $v=$ $g^{-1}[u(k x-\tilde{\omega} t)]$ nos fornece a solução:

$$
v=\left(\frac{2 k^{2}}{a}\right)^{p} \operatorname{senh}^{2 p}\left(k x-4 k^{3} p^{2} t\right),
$$

para qualquer $p$ real se $a>0$. 


\section{Obtendo modelos cosmológicos inflacionários}

O Modelo Cosmológico Padrão explica com sucesso algumas das principais propriedades observadas do nosso Universo hoje, mas infelizmente ele ainda deixa muitos problemas sem solução, como o processo de formação de estruturas em larga escala [47], ou seja, a formação de estrelas, planetas, galáxias e etc, além das anisotropias observadas pelo satélite WMAP (Wilkinson Microwave Anisotropy Probe) na Radiação Cósmica de Fundo em Microondas.

Neste sentido, a tentativa mais viável de solucionar estes problemas é apresentada no contexto da teoria de inflação, onde um campo escalar, chamado de inflaton, é responsável pela evolução do Universo primordial em um período muito curto de expansão acelerada $[1-4]$. Na Figura 6 temos a ilustração das fases de expansão cósmica do Universo tal qual o conhecemos.

Em particular, a escolha do potencial associado com o inflaton é de grande interesse, uma vez que pode proporcionar a correta dinâmica para garantir o sucesso do modelo [1]. Com base nisso torna-se necessário a busca por modelos com potenciais adequados que nos permitem encaixar parâmetros com o limite dos dados observacionais da Cosmologia 48,49.

Utilizando o método de deformação, vamos introduzir um formalismo que permite fazer a correlação entre duas dinâmicas de modelos inflacionarios sob o regime de slowroll [50 52], onde os parâmetros de inflação apresentam uma relação direta com o potencial do modelo que gera a solução.

\subsection{Considerações iniciais a respeito do regime slow-roll}

Vamos considerar a integral da ação que descreve a dinâmica de um campo escalar, $\chi(x)$, acoplada a ação de

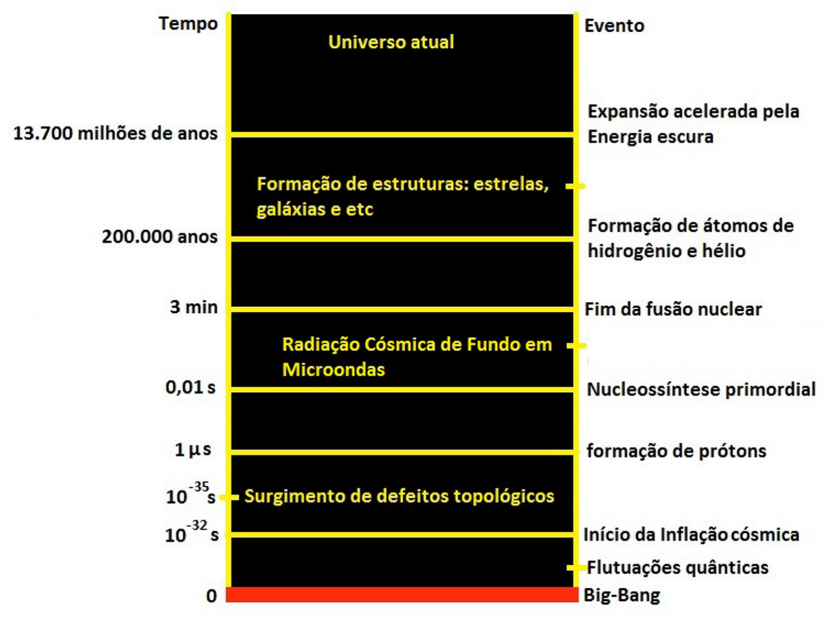

Figura 6: Processo de expansão do Universo.
Einstein-Hilbert para gravitação:

$$
S=\int d^{4} x \sqrt{-g}\left(-\frac{1}{4} R+\mathcal{L}(\chi, X)\right),
$$

onde $R$ é o escalar de curvatura, $\mathcal{L}(\chi, X)$ é a lagrangeana do modelo, $X=\partial_{\mu} \chi \partial^{\mu} \chi / 2$ é o termo cinemático e $\chi$ representa o campo de inflação.

Vamos considerar que o universo seja plano e utilizaremos a métrica de Friedmann-Robertson-Walker:

$$
d s^{2}=d t^{2}-a^{2}(t)\left(d x^{2}+d y^{2}+d z^{2}\right),
$$

onde $t$ é o tempo físico, $x, y$ and $z$ são coordenadas espaciais e $a(t)$ é o fator de escala. A partir da ação 75 e da metrica (76) obtemos as equações de movimento:

$$
H^{2}=\frac{2}{3} \rho, \quad e \quad \frac{\ddot{a}}{a}=-\frac{1}{3}(\rho+3 p),
$$

temos ainda que $H=\dot{a} / a$, com a derivada do fator de escala em relação ao tempo $t$. Se considerarmos a dinâmica padrão, descrita pela densidade de lagrangiana:

$$
\mathcal{L}=\frac{1}{2} \partial_{\mu} \chi \partial^{\mu} \chi-V(\chi),
$$

temos que a equação de continuidade pode ser escrita como:

$$
\ddot{\chi}+3 H \dot{\chi}+V_{\chi}=0,
$$

o índice $\chi$ representa a derivada em relação ao campo $\chi$. A pressão e a densidade de energia são dadas por:

$$
\rho=\frac{1}{2} \dot{\chi}^{2}+V, \quad p=\frac{1}{2} \dot{\chi}^{2}-V .
$$

Assim, podemos reescrever as Equações (77) como:

$$
H^{2}=\frac{1}{3} \dot{\chi^{2}}+\frac{2}{3} V, \text { e } \dot{H}=-\dot{\chi}^{2} .
$$

Na aproximação de slow-roll, isto é, quando $V \gg \dot{\chi}$, modelos de inflação que descrevem um universo primordial com uma densidade de energia dominada pelo potencial, $V(\chi)$, são de grande interesse. De fato, nesta aproximação, o campo do inflaton não varia muito rapidamente e podemos desprezar o termo cinético na Equação (81) para obter as equações de primeira ordem:

$$
H^{2} \approx \frac{2}{3} V(\chi), \text { e } 3 H \dot{\chi}+V_{\chi} \approx 0 .
$$

Estas equações nos mostram que a escolha do potencial nos permite aplicar limites aos parâmetros inflacionários. O número $\mathrm{N}$ de e-folds, é escrito como $N=\ln \left(a_{\text {end }} / a\right)$, onde $a_{\text {end }}$ é o fator de escala no final da inflação, pode ser obtido através da expressão:

$$
a=a_{0} \exp \left(\int_{t_{i}}^{t_{e}}\left(\frac{2}{3} V(\chi)\right)^{1 / 2} d t\right),
$$

ou equivalentimente $N=\int_{t}^{t_{\text {end }}} H d t$. 
Além disso, os parâmetros do regime de slow-roll podem ser encontrados em [53,54], e são dados por:

$$
\epsilon=\frac{1}{4}\left(\frac{V_{\chi}}{V}\right)^{2}, \quad \eta=\frac{1}{2} \frac{V_{\chi \chi}}{V} .
$$

Para estabelecer a condição de planicidade, as expressões acima devem obedecer a restrição $|\epsilon|<<1$ e $|\eta|<<1$. Portanto, podemos ver que todos os parâmetros são sensíveis à escolha do potencial.

\subsection{Deformando modelos inflacionários no regime slow-roll}

Inicialmente, consideramos que o inflaton tem sua dinâmica descrita pela densidade de lagrangiana:

$$
\mathcal{L}=\frac{1}{2} \partial_{\mu} \chi \partial^{\mu} \chi-V(\chi)
$$

onde $V(\chi)$ representa o potencial do campo $\chi$. A equação da continuidade nessa dinâmica assume a forma:

$$
\rho_{\chi}+3 H \dot{\chi}=0
$$

sabemos que

$$
H^{2}=\frac{2}{3} \rho .
$$

Elevando ao quadrado (86), nós obtemos uma util relação dada por:

$$
6 \rho \dot{\chi}^{2}=\rho_{\chi}^{2} .
$$

Vamos considerar agora uma outra dinâmica para evolução do inflaton descrita pela densidade de lagrangiana

$$
\mathcal{L}=\frac{1}{2} \partial_{\mu} \phi \partial^{\mu} \phi-\tilde{V}(\phi) .
$$

De forma similar aos resultados obtidos do modelo anterior temos:

$$
6 \tilde{\rho} \dot{\phi}^{2}=\tilde{\rho}_{\phi}^{2} .
$$

O ponto-chave desta descrição é redefinir a dinâmica do campo através da relação:

$$
\chi=f(\phi),
$$

onde $f(\phi)$ é chamada função deformadora. Como consequência direta dessa definição, podemos escrever:

$$
\dot{\phi}=\frac{\dot{\chi}}{f_{\phi}}
$$

na qual $f_{\phi}=d f / d \phi$. Usando 88 , e 90 obtemos:

$$
\frac{\tilde{\rho}_{\phi}^{2}}{\tilde{\rho}}=\frac{1}{f_{\phi}^{2}}\left(\frac{\rho_{\chi}^{2}}{\rho}\right)_{\chi=f(\phi)} .
$$

Este resultado representa uma correspondência genérica entre duas densidades de energia que descrevem dois campos escalares dinâmicos. Este é um método simples de deformar a densidade de energia para dois fluidos no mesmo background, levando em consideração que estes fluidos tenham um tensor energia-momento conservado, ou seja, desde que satisfaçam uma equação de continuidade.

A condição de slow-roll aplicada à equação de movimento do campo escalar nos permite reescrevê-lo como:

$$
3 H \dot{\chi}=-V_{\chi} .
$$

Agora

$$
6 V \dot{\chi}^{2}=V_{\chi}^{2}
$$

assim como

$$
6 \tilde{V} \dot{\phi}^{2}=\tilde{V}_{\phi}^{2}
$$

de modo que obtemos:

$$
\frac{\tilde{V}_{\phi}^{2}}{\tilde{V}}=\frac{1}{f_{\phi}^{2}}\left(\frac{V_{\chi}^{2}}{V}\right)_{\chi=f(\phi)},
$$

que representa uma correspondência direta entre dois potenciais que descrevem a dinâmica do inflaton, em uma aproximação slow-roll.

As soluções do novo modelo são obtidas de $\phi=f^{-1}(\chi)$, que é a função deformadora inversa, calculada com as soluções do modelo original. Uma implicação importante deste método está na possibilidade de obter uma descrição analítica para soluções de novos modelos de inflação, além de permitir analisar os parâmetros dessas soluções, desde que conheçamos os parâmetros para o modelo inflacionário original, sem precisar recorrer as técnicas numéricas, tornando o estudo das configurações de vácuo, antes vistas como complicadas, mais acessíveis.

O limite da condição de slow-roll deixa de ser valido para $\dot{\chi}^{2} / 2=V(\chi)$, dependendo do potencial escolhido. Temos ainda

$$
t_{\text {end }}-t_{i n i}=\int_{\chi_{i n i}}^{\chi_{e n d}} \frac{d \chi}{\sqrt{2 V}},
$$

o que leva a $t_{\text {end }}-t_{\text {ini }} \neq \tilde{t}_{\text {end }}-\tilde{t}_{\text {ini }}$, desde que (97) seja uma relação válida entre o potencial do modelo original e o potencial do modelo deformado.

\subsection{Aplicação}

Em um trabalho recente [55], partimos do modelo de inflação caótica 56 58, em que a dinâmica do campo é regida pelo potencial quadrático $V(\chi)=V_{0} \chi^{2}$. Aplicando o método de deformação podemos obter diretamente um modelo de inflação eterna [59 61], descrito pelo potencial $\tilde{V}(\phi)=\tilde{V}_{0} \phi^{p}$, onde escolhemos $p>2$, e utilizamos uma função deformadora do tipo:

$$
f(\phi)=\chi=-4 \sqrt{\frac{V_{0}}{\tilde{V}_{0}}} \frac{\phi^{-\frac{1}{2}(p-4)}}{p(p-4)},
$$

aplicada no potencial da inflação caótica, por meio da equação 97). O comportamento de ambos os regimes de inflação pode ser visto na Figura 7. 


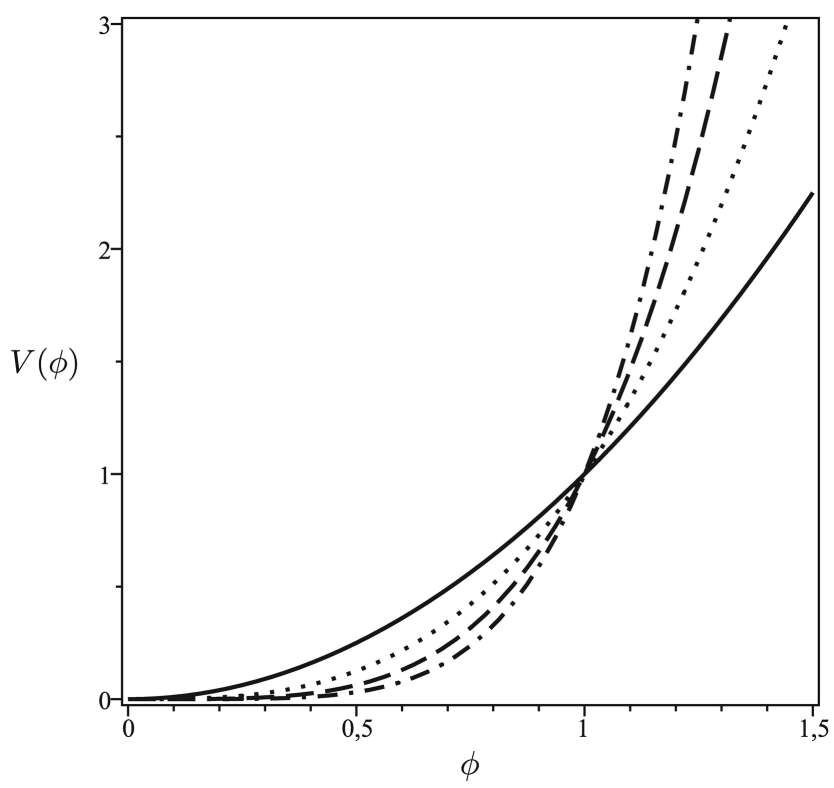

Figura 7: Gráfico do potencial do regime de inflação caótica (linha sólida) e o potencial da inflação eterna para $p=3$ (linha pontilhada), $p=4$ (linha tracejada), e $p=5$ (linha com traçoponto).

Uma vez que os potenciais são conhecidos, podemos obter os parâmetros de slow-roll. Para o potencial original temos $\epsilon=\eta=\chi^{-2}$. Aplicando o método de deformação obtemos estes parâmetros para o novo modelo deformado, dados por:

$$
\tilde{\epsilon}=\frac{p^{2}}{4 \phi^{2}} \quad e \quad \tilde{\eta}=\frac{p(p-1)}{2 \phi^{2}} .
$$

O final da inflação ocorre agora com uma escolha adicional de parâmetro $p$ para o modelo deformado, enquanto que para o modelo original apenas as condições para o campo são necessárias. O número de e-fold pode ser estimado nesse cenário como:

$$
N=\frac{1}{2}\left(\chi_{\mathrm{end}}^{2}-\chi_{\mathrm{ini}}^{2}\right)
$$

e para o modelo deformado temos o resultado dado por:

$$
\tilde{N}=\frac{1}{p}\left(\phi_{\text {end }}^{2}-\phi_{\text {ini }}^{2}\right) .
$$

Podemos observar que o caso para $p=4$ deve ser analizado separadamente. Para este valor de $p$ escrevemos o potencial como $\tilde{V}(\phi)=\lambda \phi^{4}$ e seguindo os procedimentos anteriores, a função deformadora tem a forma:

$$
f(\phi)=\chi=\sqrt{\frac{V_{0}}{\lambda}} \frac{\ln \phi}{2} .
$$

Agora, os parâmetros do regime slow-roll são escritos como:

$$
\tilde{\epsilon}=\frac{4}{\phi^{2}} \quad e \quad \tilde{\eta}=\frac{6}{\phi^{2}}
$$

Para este caso o número de e-fold é dado pelo mesmo valor $\tilde{N}$ quando $p=4$. Utilizamos funções deformadoras especiais apenas para ilustrar o procedimento, explorando resultados bem estabelecidos na literatura. No entanto, se tornarmos a escolha das funções deformadoras arbitrárias, podemos gerar novos potenciais, expandindo a gama de soluções analíticas no regime de inflação slow-roll.

\section{Considerações finais}

Neste artigo, mostramos como o método de deformação pode ser aplicado em vários cenários na teoria de campos. Alguns modelos de campos escalares, sejam aqueles descritos por potenciais polinomiais ou mesmo os que são regidos por um potencial periódico, favorecem a aplicação do método, uma vez que apresentam equações diferenciais de primeira ordem. Isso não impõe uma restrição ao método, pois podemos reduzir a ordem das equações diferenciais de ordem superior às equações diferenciais de primeira ordem, e obter soluções analíticas de interesse físico. No caso de sistemas integráveis, o método mostra-se eficaz no mapeamento de soluções de equações diferencias do tipo $\mathrm{KdV}$, que descrevem o movimento de solitons, sendo que neste contexto, utilizamos um potencial associado apenas como uma ferramenta matemática, um fator integrante que permite determinar os parâmetros dinâmicos da solução obtida pelo mapa inverso. Por fim, mostramos que o método também pode ser aplicado na construção de modelos de inflação cósmica do Universo em regime de slow-roll, levando a resultados satisfatórios e consistentes na caracterização dos parâmetros de inflação e no número de e-folds.

Os autores agradecem ao CNPq e CAPES pelo apoio.

\section{Referências}

[1] A. Guth, Phys. Rev. D 23, 347 (1981).

[2] A.D. Linde, Phys. Lett. B 108, 389 (1982).

[3] R. Crittenden e P.J. Steinhardt, Phys. Lett. B 293, 32 (1992).

[4] A.A. Starobinsky, Phys. Lett. B 117, 175 (1982).

[5] T. Padmanabham, Phys. Rep. 380, 235 (2003).

[6] R. Caldwell, R. Dave e P. Steinhardt, Phys. Rev. Lett. 80, 1582 (1998).

[7] S. Carroll, Phys. Rev. Lett 81, 3067 (1998).

[8] P. Peebles e B. Ratra, Rev. Mod. Phys. 75, 559 (2003).

[9] A. Barone, F. Esposito, C. Magee e A. Scott, Nuovo Cim. 1, 227 (1971).

[10] L. Yakushevich, Eletronic J. Theoretical Phys. 16, 97 (2007).

[11] C. Zhang, Phys. Rev. A 35, 886 (1987).

[12] C. Zhang, Phys. Rev. A 40, 2148 (1989).

[13] M. El-Batanouny, S. Burdick, K. Martine e P. Stancioff, Phys. Rev. Lett. 58, 2762 (1987).

[14] D. Bazeia, L. Losano e J. Malbouisson, Phys. Rev. D 66, 101701(R) (2002).

[15] C. Almeida, D. Bazeia, L. Losano e J. Malbouisson, Phys. Rev. D 69, 067702 (2004).

[16] D. Bazeia e L. Losano, Phys. Rev. D 73, 025016 (2006). 
[17] V.I. Afonso, D. Bazeia, M.A. Gonzalez Leon, L. Losano e J. Mateos Guilarte, Phys. Rev. D 76, 025010 (2007).

[18] D. Bazeia, M.A. Gonzalez Leon, L. Losano e J. Mateos Guilarte, Phys. Rev. D 73, 105008 (2006).

[19] D. Bazeia, L. Losano, A. Das e A. Silva, Annals Phys. 323, 1150 (2008).

[20] D. Bazeia, L. Losano, A. Das e M. dos Santos, Applied Math. Lett. 23, 681 (2010).

[21] R. Rajaramam, Solitons and Instantons (North Holland, Amsterdam, 1982).

[22] E. Bogomol'nyi, Sov. J. Nucl. Phys. 24, 449 (1976).

[23] M. Prasad and C. Sommerfield, Phys. Rev. Lett. 35, 760 (1975).

[24] C. Fred, K. Avinash e S. Uday, Supersymmetry in Quantum Mechanics (World Scientific, New Jersey, 2001).

[25] M. Shifman, A. Vainshtein e M. Voloshin, Phys. Rev. D 59, 045016 (1999).

[26] J. Morris e D. Bazeia, Phys. Rev. D 54, 5217 (1996).

[27] D. Bazeia, J. Menezes e M. Santos, Phys. Lett. B 521, 418 (2001).

[28] D. Bazeia, J. Menezes e M. Santos, Nucl. Phys. B 636, 132 (2002).

[29] M.A.M. Souza, D. Bazeia, L. Losano e R. Menezes, Latin - American Journal of Physics Education 6, 47, 2012.

[30] J. Rubistein, J. Math. Phys. 9, 258 (1970).

[31] D. Bazeia, L. Losano e R. Menezes, Phys. D 208, 236 (2005).

[32] C. Condat, R. Guyer e M. Miller, Phys. Rev. B 27, 474 (1983).

[33] D. Bazeia, L. Losano, R. Menezes e M.A.M. Souza, Europhys.Lett. 87, 21001 (2009).

[34] D. Bazeia, L. Losano, J.M.C. Malbouisson, J.R.L. Santos, Eur. Phys. J. C 71, 1767 (2011).

[35] J.S. Russell, Report of the 14th Meeting of the British Association for the Advancement of Science, British Association for the Advancement of Science (1844).

[36] D. Korteweg e G.D. Vries, Philosophical Magazine 39, 422 (1895).

[37] E. Fermi, J.R. Pasta e S.M. Ulam, Studies on Nonlinear Problems (Sci. Lab, Los Alamos, 1955).

[38] H. Flaschka, Progress, Theoret physics. 51, 703 (1974).

[39] P. Drazin e R. Johnson, Solitons: an introduction (University Press, Cambridge, 1989).

[40] J. Burgers, The nonlinear difusion equation (Reidel, Dordrecht, 1974).

[41] M. Toda, Linear and Nonlinear Waves, (Wiley, New York, 1974).

[42] N.J. Zabusky e M.D. Kruskal, Phys. Rev. Lett. 15, 240 (1965).

[43] H.A. Haus e W.S. Wong, Reviews of Modern Physics 68, 423 (1996).

[44] A. Hasegawa e M. Matsumoto, Optical Solitons in Fibers (Springer, New York, 2002).

[45] R. Hirota, Phys. Rev. Lett. 27, 1192 (1971).

[46] P.D. Lax, Commun. Pure Appl.Math. 21, 467 (1968).

[47] D.H. Lyth e A.R. Liddle, The primordial density perturbation: Cosmology, inflation and the origin of structure (Cambridge University Press, Cambridge, 2009).

[48] R. Brustein e G. Veneziano, Phys. Lett. B 329, 429 (1994).

[49] A.R. Brown, Phys. Rev. Lett. 101, 221302 (2008).
[50] A.R. Liddle, P. Parsons e J.D. Barrow, Phys. Rev. D 50, 7222 (1994).

[51] S.M. Leach e A.R. Liddle, Phys. Rev. D 68, 123508 (2003).

[52] J. Martin e C. Ringeval, JCAP 08, 009 (2006).

[53] M. Lemoine, J. Martin, e P. Peter, Lec. Notes in Phys, (Springer, Berlin, 2008).

[54] D.H. Lyth e A.R. Liddle, The primordial density perturbation: Cosmology, inflation and the origin of structure (, Cambridge, 2009).

[55] J.J. Rodrigues e M.A.M. Souza, Phys. Scr. 90, 045301 (2015).

[56] A.D. Linde, Phys. Lett. B 129, 177-181 (1983).

[57] A.S. Goncharov, A.D. Linde e V.F. Mukhanov, Int. J. Mod. Phys. A 2, 561 (1987).

[58] R. Maartens, D. Wands, B.A. Bassett e I. Heard, Phys. Rev. D 62, 041301 (2000).

[59] A.D. Linde, Mod. Phys. Lett. A 1, 81 (1986).

[60] A. Borde e A. Vilenkin, Phys. Rev. Lett. 72, 3305 (1994).

[61] A. Guth, Phys. Rept. 333, 555 (2000). 